\title{
INTRODUCTION
}

\section{Methodological issues in event-related brain potential research}

\author{
MARTIN EIMER \\ University of Cambridge, Cambridge, England
}

\begin{abstract}
Event-related brain potential (ERP) measures have become increasingly popular in experimental psychology and are often employed by researchers who are not formally trained in electrophysiology. A proper application of these measures requires knowledge about the methodological principles and practical issues that are relevant to ERP measurement, analysis, and interpretation. A brief introductory survey of different aspects of ERP methodology (recording, data processing and signal extraction, statistical analysis and interpretation of ERP effects) is presented, and the contributions collected in this special issue on ERP methodology are introduced.
\end{abstract}

In recent years, event-related brain potential (ERP) measures have become an important tool in experimental cognitive psychology. The ERP methodology has been successfully applied to the study of the processes that are involved in perception, attention, memory, language, and motor control. One reason why ERPs have become popular is that they provide continuous measures of brain activity during the time period in which the cognitive processes under investigation actually occur. In contrast, behavioral measures (response times, accuracy) usually describe discrete events that are the outcomes of such cognitive processes. The hardware and programs that are necessary for the recording and analysis of ERP waveforms are no longer only available to a small group of electrophysiologists but have already found their way into many experimental psychology labs. Amplifiers and computers are cheaper than they used to be, and commercially available software for data acquisition and analysis is much more user-friendly than those homemade programs that were used in ERP labs a few years ago.

Because the ERP methodology has come to be frequently used by researchers not formally trained in electrophysiology, there is an obvious need for practical guidelines that can help in conducting experiments employing ERP measures in a way that conforms to current methodological standards. The aim of this special issue is to address methodological issues and problems that are likely to be encountered in ERP research and to provide specific advice on how such problems can be prevented or solved. The articles address different aspects of ERP methodology-

Correspondence concerning this article should be addressed to M. Eimer, Department of Experimental Psychology, University of Cambridge, Downing St., Cambridge, CB2 3EB, England (e-mail: me209@ cam.ac.uk) for example, EEG recording, data processing, statistical analysis of ERP effects, topographical representation of brain activity, and the combination of ERPs with other brain imaging techniques. These issues will be presented in an introductory, nontechnical fashion because the primary aim of this special issue is to provide a source of information for researchers who are interested in ERP measures or already use ERPs but feel that they need to know more about the methodological side of this approach to the investigation of cognitive processes.

\section{ERP Measures in Cognitive Psychology}

ERPs reflect voltage changes in the electroencephalogram (EEG) that are related to the brain's response to specific events. These events can be external (such as the onset of a sensory stimulus) or purely internal (such as the decision to execute a response). EEG and ERPs are elicited by the synchronous activity of populations of neurons. It is assumed that they primarily reflect postsynaptic potentials that are generated at the dendrites of pyramidal cells in the cerebral cortex. To be detectable at scalp electrodes, these neurons need to be oriented in parallel and roughly perpendicular to the scalp surface (for details, see Nunez, 1981; Williamson \& Kaufman, 1990). It is important to realize that the EEG and ERP reflect only part of the total neuronal activity, so that many brain processes will not be detected on the basis of EEG and ERP recordings. It is, therefore, usually difficult to interprete null effects in ERP research. Specific experimental manipulations could leave ERP waveforms unaffected but still have an effect on brain processes that are not detectable at the scalp surface.

ERPs are extracted from epochs of EEG recordings that are time-locked to the event of interest (see below). In the resulting ERP waveforms, voltage is plotted against 
time. The positive- and negative-going deflections of the ERP are normally described with respect to their polarity (positive vs. negative: $\mathrm{P}$ vs. $\mathrm{N}$ ), their sequence, or their latency. When ERP deflections are classified with respect to polarity and sequence, the third positive deflection in an ERP waveform will be termed P3. When polarity and latency are employed, the same deflection might be described as P450 (assuming that it peaks $450 \mathrm{msec}$ after the onset of the event).

ERP measures are employed in cognitive psychology because it is assumed that ERPs are generated by brain activities that are systematically related to cognitive processes. Although this assumption might have been proved wrong, numerous studies have demonstrated that ERP deflections are indeed highly sensitive to experimental manipulations that are assumed to systematically affect different aspects of cognitive processing. By comparing the ERP waveforms that are obtained under different experimental conditions, inferences can be made with respect to the nature and properties of the underlying cognitive processes. When two experimental conditions have different effects on the ERP, it may be concluded that the cognitive processing related to these conditions (as manifested by the neural activity responsible for the ERP effects) is also different. More detailed inferences are possible when the latencies and scalp distributions of such ERP effects are taken into account (see Rugg \& Coles, 1995, for a detailed discussion of the inferential procedures employed in ERP research). It is important to note that there is not necessarily a straightforward relationship between specific cognitive processes and their neuronal sources, on the one hand, and ERP deflections like $\mathrm{P} 1, \mathrm{~N} 2$, or P3, on the other hand. The single peaks or troughs that are observed in an ERP waveform recorded at a specific scalp site may result from the summation of electrical activities generated in different areas of the brain (which may be related to different cognitive processes) that are propagated through the brain tissue to this recording site. Because ERP deflections can have multiple neural generators at different locations, the interpretation of an experimental effect on the amplitude or latency of ERP peaks and troughs can be ambiguous. To resolve this problem, the term ERP component has been introduced in order to define the features of ERP waveforms that are significant for psychological research. ERP components are assumed to be (1) generated by specific neuronal processes (Näätänen \& Picton, 1987) and (2) related to a specific cognitive function (Donchin, 1979). In the contribution by van Boxtel (1998), the difference between observationally defined ERP deflections and ERP components will be described in more detail (a brief compendium of ERP components is provided by Coles \& Rugg, 1995).

To obtain ERPs on the basis of EEG recordings and to use them as a tool for cognitive psychology, several steps in data acquisition, processing, and analysis are necessary. For each of these steps, there are important methodological principles that have to be taken into account in order to obtain reliable ERP measures and to interpret them in a meaningful way. (For general introductions into specific methodological aspects of ERP research see Coles, Gratton, \& Fabiani, 1990; Picton, 1985; Ruchkin, 1988). The following sections will give a brief survey of methodological issues in EEG recording, data processing, and signal extraction, as well as in ERP data analysis, and will introduce the contributions to this special issue in which these and related topics will be discussed in detail.

\section{EEG Recording}

The first step in ERP research is to record the EEG from experimental subjects. This is done by placing electrodes on the subjects' heads and connecting them to an amplifying system, where the amplifier output reflects the variation in voltage over time that is recorded from each scalp site (recording channel). The amplifier output is sampled on line with a specific digitization rate (measured in hertz) and is usually stored on a computer disk.

The amplifier systems used to record EEG activity usually provide different high-pass and low-pass filter settings that can be used to attenuate signal frequencies that are below and above the frequencies of interest. Lowfrequency activity (e.g., slow drifts in the EEG signal) may be attenuated by high-pass filters, whereas high frequencies (e.g., signals caused by muscle activity) can be filtered out with the help of low-pass filters. When choosing specific amplifier filter settings prior to recording, it is essential to realize that the highest measurable frequency equals half the sampling rate. Higher frequencies can lead to aliasing - that is, they are misleadingly added to the sampled waveform as increased energy at lower frequencies. (This problem is illustrated in the contribution of Srinivasan, Tucker, \& Murias, 1998.) To avoid aliasing, low-pass filter settings have to be chosen that eliminate all frequencies above the critical frequency from the EEG signal.

Recording sites are traditionally defined using the 10-20 system (Jasper, 1958), in which electrode locations are specified with respect to brain areas (frontal, temporal, parietal, occipital, and central/midline) and hemisphere (left, odd numbers; right, even numbers). For example, location T6 refers to a right temporal recording site. In high-density recordings, in which up to $128 \mathrm{elec-}$ trodes are used, numerous nonstandard sites are used in addition to the locations specified by the 10-20 system (see Srinivasan et al., 1998). The impedance between electrode and tissue should be small with respect to the input impedance of the amplifier. It is common practice to keep these impedances below $5 \mathrm{k} \Omega$.

The number and locations of recording sites used in an experiment depends on the type of research question investigated. When the aim is to obtain realistic scalp topographies of brain activities (e.g., in order to make inferences about the localization of neural generator processes; see below), densely spaced electrode arrays are required. This will be demonstrated in the contributions of Srinivasan et al. (1998) and Bauer (1998). Bauer de- 
scribes methodological principles that are relevant for studies of the topography of slow brain potential changes. These include aspects of EEG recording, spatial sampling, and imaging. Srinivasan et al. demonstrate that, for topographical mapping, analysis, and interpretation of ERP activity, adequate spatial sampling is as important as adequate temporal sampling and conclude that topographical maps that are based on an insufficient number of channels may introduce significant spatial sampling errors. It should be noted that this argument holds for topographical mapping studies but not for experiments in which the primary aim is to obtain ERP markers of differential cognitive processing by studying the magnitude and the time course of experimental effects at single recording sites.

The voltages obtained for each recording channel are not pure measures of the activity obtained at this electrode but rather differences in voltage between that site (active electrode) and another (reference) electrode. Because of that, there should be relatively little electrical activity at reference electrodes. To obtain reliable EEG and ERP waveforms, an appropriate choice of reference electrodes is extremely important. With common reference, all active electrodes are connected to the same reference site (which could be a single electrode located at the tip of the nose or a pair of electrodes linked together, as in the "linked mastoid" reference). To obtain an average reference, the mean voltage across all electrodes is computed and then subtracted from the voltage obtained at each recording site. In the contribution by Dien (1998), common reference and average reference techniques are compared. In addition, it is demonstrated how the choice of different reference sites can affect scalp topographies of ERP waveforms as well as statistical analyses of ERP effects.

\section{Data Processing and Signal Extraction}

After EEG recording, the sampled data have to be further processed in order to obtain reliable event-related brain potential waveforms. The processing of raw EEG data includes artifact detection and its rejection or correction, digital filtering, and the extraction of ERP waveforms from the background EEG.

One important step in data processing is to identify and eliminate signals that originate from extracranial sources. Artifacts caused by muscle activity or bodily movements usually show larger amplitudes or higher frequencies as EEG signals and can therefore be detected quite easily in the EEG recordings. Other important sources of artifacts are eye movements or blinks. Since these movements produce electrical activity that will be propagated across the different recording channels, eye movement or blink activity is usually monitored at special electrode sites. Experimental trials in which eye movements or blinks occurred may be eliminated from further analysis. Alternatively, eye movement correction procedures can be employed that propose to reduce or even eliminate ocular artifacts from the recording channels. Gratton (1998) pro- vides a comprehensive introduction to the problem of ocular artifacts as well as a description and evaluation of different correction procedures. In addition, a number of practical guidelines on how to deal with ocular artifacts are proposed.

Digital filtering procedures can be employed to eliminate or attenuate all irrelevant (high or low) frequencies in the EEG waveform. Although this could, in principle, also be accomplished by choosing appropriately narrow amplifier filter settings (analog filtering; see above), digital filters are widely in use in the processing of sampled EEG data. One reason for this is that digital filters are often superior to analog filters with respect to preserving temporal relationships among signals. Another reason is that, in contrast to analog filtering, the input data can (and should) be preserved, so that sequential filtering operations will not cause an irreversible transformation of the data. The contribution by Nitschke, Miller, and Cook (1998) describes applications of digital filtering in ERP research. A general framework for the description of digital filters is provided, several filtering techniques used in ERP research are presented, and it is shown that many of these filters may not be entirely adequate.

The last step in signal processing consists of the extraction of the ERP waveform from the residual, spontaneous, or background EEG. The amplitude of the ERP waveform (signal) is small in comparison with the amplitude of the EEG activity that is not time-locked to an event (noise). To improve this signal-to-noise ratio and to obtain ERP waveforms that are largely unaffected by noise activity, several EEG recording epochs that are time-locked to repetitions of the same event are combined. This is usually done by averaging. The amplitude values for each time point (measured relative to the onset of the event) are averaged in order to obtain a single value for that time point, which results in a time series of averaged values that constitutes the event-related potential. Because averaged EEG activity that is not time-locked to the event will approach zero, this procedure removes the noise without affecting the signal.

Although widely in use, the averaging procedure has several problems that should be acknowledged. The most important problem is that averaged ERP waveforms will not neccessarily be equivalent to the ERPs elicited in individual trials. When there is systematic variability in the amplitudes or latencies of specific ERP deflections between trials, the averaged waveforms may seriously misrepresent the actual amplitudes or latencies for any individual trial. Several procedures have been proposed to deal with the problem of temporal variability between trials. One widely used approach is an adaptive filtering method devised by Woody (1967), which will be described and evaluated in the contributions of van Boxtel (1998) and of Hoormann, Falkenstein, Schwarzenau, and Hohnsbein (1998). In the paper by Yabe (1998), the median method is described as an alternative to the averaging procedure. Instead of computing the arithmetic mean of single-trial amplitudes at each time point, the median value 
is used to determine the ERP waveform. It is demonstrated that the median method can deal effectively with temporal variability of signals between trials as well as with infrequent artifacts and may thus be an interesting alternative to the averaging procedure.

Another problem related to between-trial signal variability is especially relevant for topographical studies of brain activity, in which the distribution of ERP peaks or troughs across different scalp sites is of specific interest. In such studies, changes in the topography of an ERP peak between conditions (or subject groups) are often considered to indicate important functional differences. However, variability in these scalp distributions between single trials is usually not assessed. A nonparametric bootstrapping method for determining the reliability of the locations of maxima in topographical maps of brain activity for individual subjects is described in the contribution of Fabiani, Gratton, Corballis, Cheng, and Friedman (1998).

\section{Data Analysis and Interpretation}

After ERP waveforms have been computed on the basis of EEG recordings, the next step is to analyze the effects of the experimental variables on these ERPs. A straightforward way to do this is to measure the amplitudes or latencies of specific ERP deflections (peaks or troughs) separately for all experimental conditions and to analyze these values with appropriate statistical procedures (e.g., with repeated measures analyses of variance, paired $t$ tests). Several simple procedures for quantification and statistical testing of ERP amplitude differences between conditions (peak analysis, analysis of mean amplitudes obtained within predefined measurement windows, analysis of difference waveforms) are discussed in the paper by Hoormann et al. (1998). These authors also propose a simple new method (window analysis) for quantifying and testing ERP effects. In addition to differences in ERP amplitude, differences in the latencies of ERP deflections can also be of specific interest to cognitive psychologists. In the contribution of Schwarzenau, Falkenstein, Hoormann, and Hohnsbein (1998), a new method for determining the onset latency of the lateralized readiness potential (LRP) is introduced. Different methods for detecting the onset of electromyographic (EMG) responses are described and evaluated in the contribution of van Boxtel (1998).

As described above, specific ERP deflections do not necessarily correspond to ERP components, because the voltage measured at any site may be a result of the overlapping activities of different spatially and functionally distinct generator processes. The simple methods for the quantification and testing of ERP effects described above may thus be of limited value with respect to the identification of ERP components. Two alternative ways of extracting ERP components have been proposed-a physiological approach and a functional approach (see Coles \& Rugg, 1995, for a further discussion of this issue). According to the functional approach, ERP components are related to specific cognitive functions that are performed in a given experimental situation. One way to identify ERP components, thus, is to systematically analyze covariation patterns in ERP waveforms that are obtained under different experimental conditions. A widely used method for identifying common sources of variance in ERP data is principal component analysis (PCA). This method is introduced and described in detail in the contribution of van Boxtel (1998).

According to the physiological approach, ERP components are elicited by unique intracranial sources. To identify ERP components, the generator processes responsible for ERP deflections that are observed at the scalp surface have to be identified. Source analysis techniques have been proposed in which such generator processes are inferred on the basis of the topographical information obtained by EEG recordings with dense electrode arrays (cf. Scherg, 1990). Although these techniques may provide important cues for the localization of the brain processes that are responsible for ERPs, they are nevertheless subject to the inverse problem: It is in principle not possible to uniquely specify underlying neural generators exclusively on the basis of topographical maps that are generated from scalp surface recordings. However, by combining ERP recordings with other neuroimaging methods (such as positron emission tomography [PET] or functional magnetic resonance imaging [fMRI], additional information can be obtained that will help to localize intracranial sources of ERP activity. This approach is described in the contribution of Mangun, Hopfinger, and Heinze (1998), who show that the integration of ERP, PET, and fMRI measures can provide constraints for determining the loci of ERP generator processes. These authors discuss visual-spatial attention as a case study for their integrative approach. Because of the increasing availability of modern brain imaging techniques, the combination of ERP measures with these methods will become even more important in the next decade.

\section{Application of ERP Measures in Psychological Research}

Although the principles summarized above and the topics covered by the articles assembled in this special issue may provide an introduction to the general methodology of ERP research, specific research questions that are related to specific ERP components usually give rise to rather specific methodological considerations. In the contribution by Schröger (1998), methodological and conceptual aspects related to the recording, analysis, and interpretation of the mismatch negativity (MMN) will be discussed. The MMN is elicited by changes in repetitive auditory stimulation and has been employed in numerous studies that investigate auditory sensory memory. Another ERP component that is of specific interest to cognitive psychologists is the LRP that is assumed to reflect central motor activation processes. In the contribution by Eimer (1998), the methods for deriving the LRP on the basis of EEG recordings obtained over the left and right motor cortices are described, and several conceptual and methodological issues that need to be considered in 
order to interpret the LRP as a valid index of central response activation processes are discussed.

ERP measures are not only used in experimental psychology but have also been frequently applied in psychiatric and neuropsychological contexts. Numerous ERP studies have been conducted with patients suffering from psychiatric or neurological disorders. ERP research with neurological patients may aid the understanding of the nature and effects of specific neurological diseases. In addition, it can also provide additional insights into the functional significance and neural basis of specific ERP components by comparing ERPs obtained for patients who have different brain lesions with ERPs recorded for healthy control subjects. The contribution by Swaab (1998) discusses methodological principles that are relevant for ERP studies with patient populations and illustrates the use of ERPs in a specific field of neuropsychological research (the study of aphasia).

\section{REFERENCES}

BAUER, H. (1998). Slow potential topography. Behavior Research Methods, Instruments, \& Computers, 30, 20-33.

Coles, M. G. H., Gratton, G., \& Fabiani, M. (1990). Event-related potentials. In J. T. Cacioppo \& L. G. Tassinary (Eds.), Principles of psychophysiology: Physical, social, and inferential elements (pp. 413455). Cambridge: Cambridge University Press.

COLES, M. G. H., \& RUGG, M. D. (1995). Event-related brain potentials: An introduction. In M. D. Rugg \& M. G. H. Coles (Eds.), Electrophysiology of mind. Event-related brain potentials and cognition (pp. 1-26). New York: Oxford University Press.

DIEN, J. (1998). Issues in the application of the average reference: Review, critiques, and recommendations. Behavior Research Methods, Instruments, \& Computers, 30, 34-43.

DonCHIN, E. (1979). Event-related brain potentials: A tool in the study of human information processing. In H. Begleiter (Ed.), Evoked potentials and behavior (pp. 13-75). New York: Plenum.

EIMER, M. (1998). The lateralized readiness potential as an on-line measure of central response activation processes. Behavior Research Methods, Instruments, \& Computers, 30, 146-156.

Fabiani, M., Gratton, G., Corballis, P. M., Cheng, J., \& FriedMAN, D. (1998). Bootstrap assessment of the reliability of maxima in surface maps of brain activity of individual subjects derived with electrophysiological and optical methods. Behavior Research Methods, Instruments, \& Computers, 30, 78-86.

GRATTON, G. (1998). Dealing with artifacts: The EOG contamination of the event-related brain potential. Behavior Research Methods, Instruments, \& Computers, 30, 44-53.

Hoormann, J., Falkenstein, M., Schwarzenau, P., \& Hohnsbein, J. (1998). Methods for the quantification and statistical testing of ERP differences across conditions. Behavior Research Methods, Instruments, \& Computers, 30, 103-109.

JASPER, H. H. (1958). The ten-twenty electrode system of the Interna- tional Federation. Electroencephalography \& Clinical Neurophysiology, 10, 371-375.

Mangun, G. R., Hopfinger, J. B., \& Heinze, H.-J. (1998). Integrating electrophysiology and neuroimaging in the study of human cognition. Behavior Research Methods, Instruments, \& Computers, 30, 118-130.

NÄÄTÄNEN, R., \& PICTON, T. W. (1987). The N1 wave of the human electric and magnetic response to sound: A review and an analysis of the component structure. Psychophysiology, 24, 375-425.

NitsChKe, J. B., Miller, G. A., \& COOK, E. W., III (1998). Digital filtering in EEG/ERP analysis: Some technical and empirical comparisons. Behavior Research Methods, Instruments, \& Computers, 30, 54-67.

NUNEZ, P. L. (1981). Electrical fields of the brain: The neurophysics of $E E G$. New York: Oxford University Press.

PICTON, T. W. (1985). The recording and measurement of evoked potentials. In A. M. Halliday, S. R. Butler, \& R. Paul (Eds.), A textbook of clinical neurophysiology (pp. 23-40). New York: Wiley.

RUCHKIN, D. M. (1988). Measurement of event-related potentials: Signal extraction. In T. W. Picton (Ed.), Human event-related potentials. EEG handbook (Vol. 3, pp. 7-43). Amsterdam: Elsevier.

RugG, M. D., \& Coles, M. G. H. (1995). The ERP and cognitive psychology: Conceptual issues. In M. D. Rugg \& M. G. H. Coles (Eds.), Electrophysiology of mind: Event-related brain potentials and cognition (pp. 27-39). New York: Oxford University Press.

SCHERG, M. (1990). Fundamentals of dipole source potential analysis. In F. Grandori, M. Hoke, \& G. L. Romani (Eds.), Auditory evoked magnetic fields and electric potentials. Advances in audiology (Vol. 6, pp. 40-69). Basel: Karger.

SCHRÖGER, E. (1998). Measurement and interpretation of the mismatch negativity. Behavior Research Methods, Instruments, \& Computers, 30, 131-145.

Schwarzenau, P., Falkenstein, M., Hoormann, J., \& Hohnsbein, J. (1998). A new method for the estimation of the onset of the lateralized readiness potential (LRP). Behavior Research Methods, Insiruments, \& Computers, 30, 110-117.

Srinivasan, R., TUCKer, D. M., \& MurIas, M. (1998). Estimating the spatial Nyquist of the human EEG. Behavior Research Methods, Instruments, \& Computers, 30, 8-19.

SWAAB, T. Y. (1998). Event-related potentials in cognitive neuropsychology: Methodological considerations and an example from studies of aphasia. Behavior Research Methods, Instruments, \& Computers, 30, 157-170.

VAN BOXTEL, G. J. M. (1998). Computational and statistical methods for analyzing event-related potential data. Behavior Research Methods, Instruments, \& Computers, 30, 87-102.

Williamson, S. J., \& KaUfMaN, L. (1990). Theory of neuroelectric and neuromagnetic fields. In F. Grandori, M. Hoke, \& G. L. Romani (Eds.), Auditory evoked magnetic fields and electric potentials. Advances in audiology (Vol. 6, pp. 1-39). Basel: Karger.

WooDY, C. D. (1967). Characterization of an adaptive filter for the analysis of variable latency neuroelectric signals. Medical \& Biological Engineering, 5, 539-553.

YABE, H. (1998). Median method for eliminating in frequent artifacts and identifying the signals blurred by latency jitter and uncertain occurrence. Behavior Research Methods, Instruments, \& Computers, 30, 68-77. 